\title{
Comment on: Langerhans cell histiocytosis with BRAF p.N486_P490del or MAP2K1 p.K57_G61del treated by the MEK inhibitor trametinib
}

\author{
Paige Vicenzi ${ }^{1}$ and Anish Ray ${ }^{2}$ \\ ${ }^{1}$ UNTHSC \\ ${ }^{2}$ Cook Children's Health Care System
}

October 8, 2020

Comment on: Langerhans cell histiocytosis with BRAF p.N486_P490del or MAP2K1 p.K57_G61del treated by the MEK inhibitor trametinib

${ }^{1}$ Paige Vicenzi, OMS-IV, ${ }^{2}$ Anish Ray, MD

${ }^{1}$ Texas College of Osteopathic Medicine, University of North Texas Health Science Center

${ }^{2}$ Department of Pediatric Hematology/Oncology, Cook Children's Health Care System

Corresponding Author:

Anish Ray, MD

1500 Cooper St., 5th floor,

Fort Worth, TX 76104

Phone: 425-205-0926

Anish.Ray@CookChildrens.org

Word Count: 513

Number of Tables: 0

Number of Figures: 0

Running Title: Langerhans cell histiocytosis treated by trametinib

Keywords: Langerhans cell histiocytosis, MAP2K1, trametinib, pediatric

The authors have no financial support or conflicts of interest.

Langerhans cell histiocytosis (LCH) is a rare but heterogenous myeloid malignancy. The discovery of mitogen-activated protein kinase (MAPK) pathway activating mutations as key oncogenic drivers offered only equivocal implications at best; the promise of targeted therapy was often eclipsed by a more severe clinical course, risk organ involvement, poorer response to standard therapy, and higher risk of relapse. ${ }^{1}$ There is, however, mounting evidence in support of MAPK pathway inhibition for patients with BRAF V600E mutations. A recent report outlines rapid and durable response of relapsed, multisystemic LCH with either BRAF p.N486_P490 or MAP2K1 p.K57_G61 deletion to MEK inhibitor trametinib. ${ }^{2}$ Two of the three patients achieved nonactive disease, including a 2-year-old male with MAP2K1 deletion who, despite reports attributing trametinib resistance to MAP2K1 mutations ${ }^{3}$, continues to thrive. We take this opportunity 
to describe an analogous experience treating a relapsed LCH patient with trametinib at Cook Children's Medical Center from early 2020 to present.

Our patient is a 4-year-old male who presented in March 2017 with new onset central diabetes insipidus (DI) and skin rash; skin biopsy provided diagnosis of LCH, but skeletal survey was negative for bone involvement. He was treated with twelve cycles of cytarabine $\left(100\right.$ milligram $(\mathrm{mg}) / \mathrm{m}^{2}$ intravenous daily for five days, every four weeks) and DDAVP for DI. At the completion of cytarabine, a second skin biopsy revealed recurrence of $\mathrm{LCH}$, which warranted treatment with hydroxyurea $(20 \mathrm{mg} /$ kilogram $(\mathrm{Kg})$ daily $)$ and methotrexate $(2.5 \mathrm{mg}$ at $0.12 \mathrm{mg} / \mathrm{Kg}$ twice a week). This was continued for 52 weeks despite a brief interruption of methotrexate due to dermatitis. Three months following completion of this therapy, brain MRI revealed a $7 \mathrm{~mm}$ lesion of the skull. Curettage by neurosurgery confirmed relapse of LCH in January 2020. Genetic testing of this sample was negative for $B R A F$ mutation, but positive for a mutation in the MAP2K1 gene, specifically a point mutation resulting in a substitution of Q56P. Shortly after his biopsy, the patient developed a soft tissue swelling on his skull. Due to these results and his multiple relapses, the patient was started on trametinib ( $2.5 \mathrm{mg}$ daily) in February 2020 with rapid resolution of skull swelling and transient but dramatic reduction of his desmopressin dose from $3.2 \mathrm{mg}$ twice a day to $0.2 \mathrm{mg}$ twice a day. He has not experienced toxicity and continues to tolerate the drug well.

Though trametinib presents a promising treatment for high-risk, relapsed LCH, it is not without limitations. In 2020, we also treated a 15-year-old male with relapsed LCH and BRAF V600E with trametinib monotherapy. Due to skin rash (Grade II), the patient became noncompliant. Despite stopping altogether after a month of treatment, he has yet to experience disease recurrence. But as stated in the aforementioned report, sufficient dose and treatment length to attain MAPK pathway suppression merits further investigation. In our similar experience treating a young child with multisystemic LCH and MAP2K1 mutation, we remain encouraged that MEK inhibition via trametinib monotherapy is a viable treatment option. In the context of genomic landscaping, we hope to incite further exploration of targeted therapy, and consequently, greater consensus on LCH management.

\section{Conflict of Interest}

The authors declare that there is no conflict of interest.

\section{References}

1. Héritier S, Emile J-F, Barkaoui M-A, et al. BRAF Mutation Correlates With High-Risk Langerhans Cell Histiocytosis and Increased Resistance to First-Line Therapy. Journal of clinical oncology : official journal of the American Society of Clinical Oncology.2016;34(25):3023-3030.

2. Messinger YH, Bostrom BC, Olson DR, Gossai NP, Miller LH, Richards MK. Langerhans cell histiocytosis with BRAF p.N486_P490del or MAP2K1 p.K57_G61del treated by the MEK inhibitor trametinib. Pediatric Blood \& Cancer. 2020;n/a(n/a):e28712.

3. Azorsa DO, Lee DW, Wai DH, et al. Clinical resistance associated with a novel MAP2K1 mutation in a patient with Langerhans cell histiocytosis.Pediatric Blood $\mathcal{E}$ Cancer. 2018;65(9):e27237. 